\title{
Study on Vibration Reduction Method for a Subway Station in Soft Ground
}

\author{
Xian-Feng Ma, ${ }^{1,2}$ Ning-Ning Feng, ${ }^{1}$ Guo-Bo Wang, ${ }^{3}$ and Xu Fang ${ }^{1}$ \\ ${ }^{1}$ Department of Geotechnical Engineering, Tongji University, Shanghai 200092, China \\ ${ }^{2}$ Key Laboratory of Geotechnical and Underground Engineering of Ministry of Education, Tongji University, Shanghai 200092, China \\ ${ }^{3}$ Hubei Key Laboratory of Roadway Bridge \& Structure Engineer, Wuhan University of Technology, Wuhan 430070, China
}

Correspondence should be addressed to Guo-Bo Wang; wgb16790604@126.com

Received 28 July 2017; Accepted 23 October 2017; Published 14 November 2017

Academic Editor: Xing Ma

Copyright (C) 2017 Xian-Feng Ma et al. This is an open access article distributed under the Creative Commons Attribution License, which permits unrestricted use, distribution, and reproduction in any medium, provided the original work is properly cited.

\begin{abstract}
With the rapid development of metro system in urban areas, vibration and its impact on adjacent structures caused by metro operation have drawn much attention of researches and worries relating to it have risen. This paper analyzed the vibration attenuation and the environment impact by a case study of a subway station in soft ground with adjacent laboratory building. A method of setting a compound separation barrier surrounding the station is checked and different materials used in the barrier have been tried and tested through numerical analysis. Key parameters of the material and the effects of vibration reduction are studied with the purpose that similar methodology and findings can be referenced in future practices.
\end{abstract}

\section{Introduction}

In the operation of large-scale metro system in modern cities around the world, vibration caused by running trains of metro system and its impact on environment have become increasingly problematic, especially in big cities with densely built structures and overcrowded population in both developed countries and emerging economies. Attentions of scholars in many countries have been drawn on this issue and vibration separation and reduction measures to tackle the subway vibration impact have been studied, among which barriers are usually assumed to be one of the effective ways.

Since 1960s, scholars including Barkan et al. [1-9] carried out researches on vibration isolation by barriers and proposed preliminary strategies relating to it. Qiu et al. [10] have also conducted relevant researches since 1998 in terms of efficiencies and environmental impacts of vibration barrier. Notwithstanding the above efforts, vibration barrier theory and some findings remain conditionally applicable, partly due to the complexity of the boundary value problems on site. Nowadays, researches on vibration isolation by barriers have been mainly based on numerical methods such as finite element method and boundary element method, as well as indoor model tests or in situ tests. Useful findings have been obtained and some results have also been proposed on vibration isolation using barrier, but many of them still fall short of application on site due to insufficient calculation conditions or incomplete test cases on many occasions. On the other hand, in most of these researches, single material was employed in vibration isolation, while there are very few researches on the effect of using compound material in vibration isolation though there would be enough chances for compound material to be beneficial both technically and economically in vibration separation and reduction.

In this paper, a case study on vibration reduction methodology for a subway station in Shanghai is reported, aiming to answer the need of reducing the vibration impact on a physics laboratory building in close proximity to the station. The method includes a vibration separation trench surrounding the station structure and isolation materials that would be filled into the trench. The effects of different isolation materials were checked using numerical analysis and the results helped to guide the practice of vibration reduction for subway stations in Shanghai soft ground. 


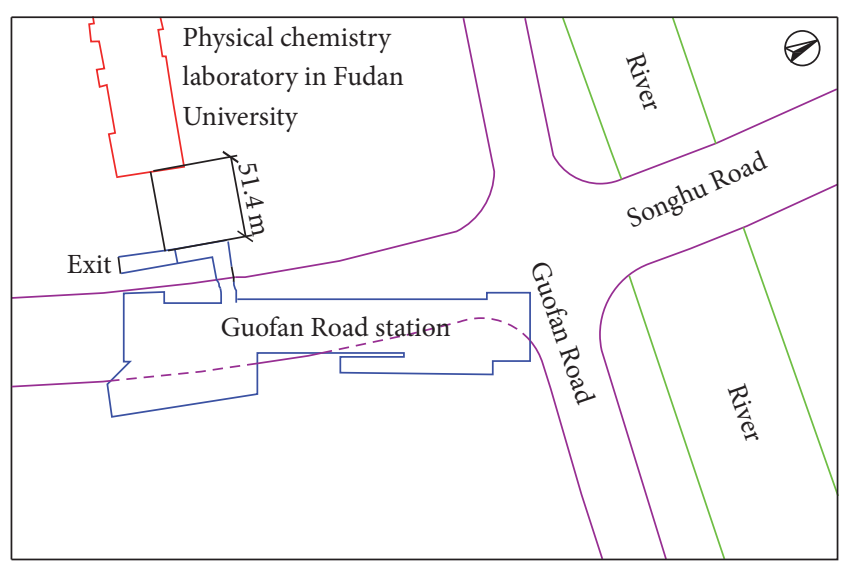

FIGURE 1: Layout of Guofan Road station and the adjacent building.

\section{Subway Station Studied in This Paper}

The station studied in this paper is Guofan Road subway station, which is under construction in phase two project of Shanghai Metro Line number 10. Due to the restrictions of the planning and site selection, Guofan Road station is set to be located at Songhu Road, and the west side is adjacent to New Jiangwan Campus of Fudan University, as shown in Figure 1. Inside the New Jiangwan Campus, there is a State Key Physical Chemistry Laboratory which is about $50 \mathrm{~m}$ away from the subway station. With consideration of strict vibration requirements for the surrounding environment needed for laboratory operation, measures have to be taken to ensure that there will be no adverse effect on daily operation of the laboratory after the station would be put into service. So the main issue is how to separate and reduce the vibration caused by the metro operation in the station.

\section{Numerical Study on Vibration Separation Scheme}

3.1. Comparison and Selection of Vibration Reduction Materials. After preliminary studies, it is decided to build a trench filled with soft materials surrounding the subway station wall to separate the vibration for the laboratory. Investigation on applicable materials that can be used in vibration reduction shows the following list: sand cushion, soil sandbag, STC reinforced sand, foam polyurethane, polymer, and foam concrete. Taking durability and cost aspect into consideration as well as the effect of reducing vibration, foam concrete and yellow sand were selected as the filling materials used in the trench separating Guofan Road station from surrounding ground. Several combinations of the two materials were studied in this research to achieve the required vibration reduction effect, while the cost could be controlled at the same time.

3.2. Barrier Filling Scheme for the Trench Surrounding the Station. In order to work out a reasonable filling scheme for the barrier to reduce and separate the vibration from the Guofan Road station, three alternatives were proposed for further

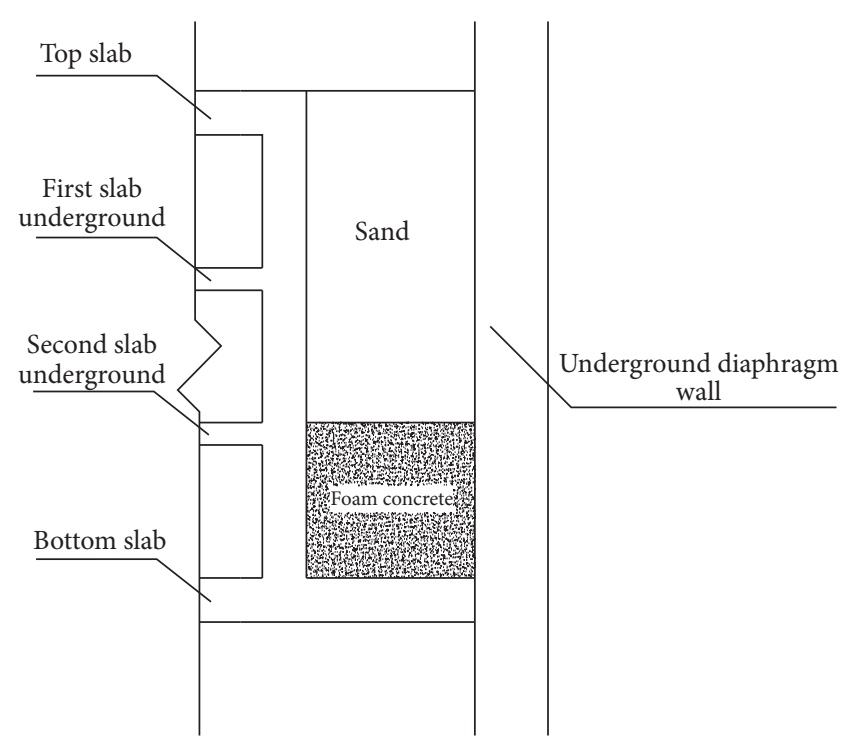

Figure 2: Sketch map of Scheme 1.

study, and selection was made based on comparison among them considering the feasibility of construction, economic aspect, and the effect of vibration isolation and reduction. The following part describes the three filling schemes for vibration reduction materials.

(1) Scheme 1. The space in trench between the side wall of the station structure and the diaphragm retaining wall will be filled by foam concrete at the depth of the third level of the station structure, and the rest space in the trench will be filled with sand, as shown in Figure 2.

(2) Scheme 2. The space in the trench at the depth of the second and third levels will be filled with foam concrete and the rest is filled with sand.

(3) Scheme 3. The space between the station structure's external walls and the diaphragm wall are all filled with sand.

During the process of numerical simulation, in order to acquire a better comparison among potential measures, some other schemes were added for further analysis. The added schemes include the following alternatives: all of the trench filled with foam concrete (Scheme 4); all trench filled with normal concrete (Scheme 5); and an open-air trench without any filling material (Scheme 6). Considering that the range of elasticity modulus of foam concrete is within $40 \mathrm{Mpa}$ $1200 \mathrm{Mpa}$ [11-13], the elasticity modulus is taken as parameters together with Poisson ration and the damping ration of foam concrete, for the purpose of carrying out parametric study to find out the more effective case. Each group of the parameters makes up one calculation case and thus there are six calculation cases altogether, and each of them includes six schemes of filling barrier material described above. So comparison between cases will help to decide which kind of foam concrete is more effective, while comparison between 
TABLE 1: Calculation cases.

\begin{tabular}{lcccccc}
\hline Cases & 1 & 2 & 3 & 4 & 5 & 6 \\
\hline Parameters of foam concrete & & & & & & \\
$E(\mathrm{Mpa})$ & 40 & 40 & 40 & 40 & 40 & 1000 \\
$c$ & 0.05 & 0.5 & 0.95 & 0.05 & 0.05 & 0.05 \\
$v$ & 0.21 & 0.21 & 0.21 & 0.31 & 0.48 & 0.21 \\
\hline
\end{tabular}

each scheme of a specific case is useful to decide which scheme is more effective and economical at the same time. Table 1 shows parameters in calculation cases.

3.3. Numerical Calculation Parameters. The excavation depth of the Guofan Road station is about $23.32 \mathrm{~m}$ with the width of about $21.5 \mathrm{~m}$ and the length of about $156.5 \mathrm{~m}$. The thickness of the underground diaphragm wall is $1 \mathrm{~m}$ and its depth is $41.5 \mathrm{~m}$. The soil layers encountered during the excavation of the station are as follows: layer (1)1, layer (2)1, layer (3), layer (4), and layer (5), whose names are officially designated according to the sequence they are encountered from the surface to a deeper layer. Ground at the bottom level of the station is in the (5) 2 layer. According to the ground investigation report and the shear wave velocity of Shanghai soil layer in Table 3, the physical and mechanical parameters of soil layer in Shanghai can be obtained taking into consideration (1), as is shown in Table 2. The physical and mechanical parameters of C30 concrete which is used in the subway station, as well as the parameters of foam concrete, can be referred to Qiu-Huan et al's paper [14]. The parameters of medium sand refer to the literature $[15,16]$. The constitutive relationship of soil, foam concrete, and medium sand is adopted as Mohr-Coulomb model, and the parameters are given in Table 3.

$$
\begin{aligned}
\lambda & =\frac{\nu E}{(1+\nu)(1-2 \nu)}, \\
\mu & =G=\frac{E}{2(1+\nu)}, \\
E & =\frac{\nu(3 \lambda+2 \nu)}{\lambda+\nu}, \\
\nu & =\frac{\lambda}{2(\lambda+\nu)}, \\
C_{P} & =\sqrt{\frac{\lambda+2 G}{\rho}}, \\
C_{S} & =\sqrt{\frac{G}{\rho}}=\sqrt{\frac{E}{2(1+\nu) \rho}} .
\end{aligned}
$$

It is well known that, in analyzing dynamic problems, in order to eliminate the adverse effect of the reflection of the vibration wave on the boundary, the artificial boundary condition should be set at the boundary. The boundary conditions for this numerical calculation are viscous-spring artificial boundaries, which can be referred to $\mathrm{Gu}$ et al.s [17].

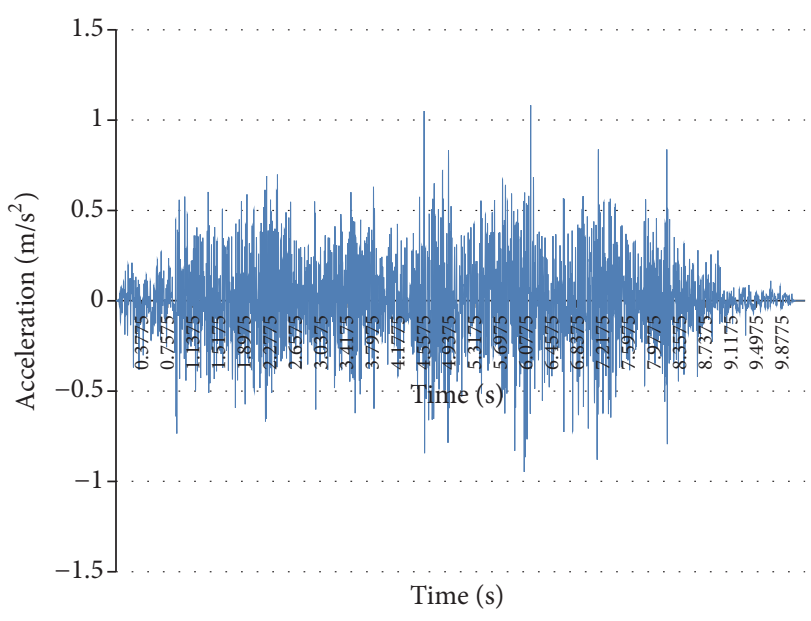

Figure 3: Acceleration time histogram.

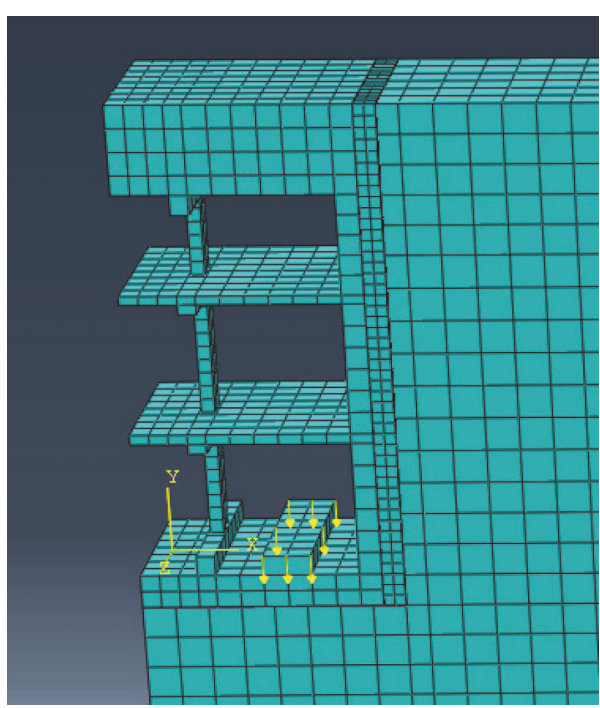

FIGURE 4: Details of station and location of acceleration.

3.4. Three-Dimensional Model, Applied Excitation, and Monitoring Nodes. The commercial software ABAQUS is used in this numerical calculation. Considering that the subway vibration is very small compared to the earthquake wave and thus no large relative displacement could be caused between soil and station structure, soil and diaphragm wall, and filling materials and surrounding structures, so these interfaces were assumed to be in displacement coordination condition, namely, binding constraints in the ABAQUS. Station, soil, diaphragm wall, and filling materials are modeled by solid element C3D8 in the software. The viscous-spring artificial boundary is applied to the boundary of the model, and the measured acceleration time-history at the rail ballast of a subway station in Shanghai was set to be the input excitation on the ballast (Figure 3). Based on the above model, filtering and spectrum analyses were performed. The excitation is applied by the boundary condition, and the applied position is shown in Figure 4. The finite element three-dimensional model with vertical depth of $60 \mathrm{~m}$, horizontal length of 
TABLE 2: Parameters of Shanghai soil.

\begin{tabular}{|c|c|c|c|c|c|c|c|}
\hline Soil layers & Depth (m) & $C_{S}(\mathrm{~m} / \mathrm{s})$ & $E_{d}(\mathrm{MPa})$ & $\rho\left(\mathrm{kg} / \mathrm{m}^{3}\right)$ & $v$ & $c(\mathrm{kPa})$ & $\varphi\left(^{\circ}\right)$ \\
\hline (1)1, (2)1 & 6.9 & 101.80 & 52.43 & 1902.20 & 0.33 & 21 & 16 \\
\hline (3), (4) & 10.6 & 136.22 & 84.64 & 1689.43 & 0.35 & 13 & 12.5 \\
\hline (5) 1 & 4.8 & 178.65 & 155 & 1710 & 0.42 & 16 & 12.5 \\
\hline (5) 2 & 13.9 & 211.21 & 218 & 1810 & 0.35 & 4 & 30.5 \\
\hline (5) 3, (5) 4 & 4.1 & 275.94 & 379.68 & 1888.78 & 0.32 & 33 & 17 \\
\hline (7)1, (7) 2 & 4.8 & 335.61 & 556.5 & 1930 & 0.28 & 8 & 32.5 \\
\hline (8)1 & 8.9 & 352.06 & 589 & 1800 & 0.32 & 23 & 18 \\
\hline (8) 2 & 6 & 376.39 & 685.1 & 1860 & 0.3 & 24 & 18.5 \\
\hline
\end{tabular}

Note. $C_{S}=$ shear wave velocity; $E_{d}=$ dynamic modulus of elasticity; $\rho=$ density; $\nu=$ Poisson ratio; $c=$ cohesion; $\varphi\left({ }^{\circ}\right)=$ inner friction angle.

TABLE 3: Parameter of foam concrete, medium sand, and C30 concrete.

\begin{tabular}{lccccc}
\hline Materials & $E(\mathrm{MPa})$ & $\rho\left(\mathrm{kg} / \mathrm{m}^{3}\right)$ & $v$ & $c(\mathrm{kPa})$ & \multicolumn{1}{c}{} \\
\hline C30 concrete & 30000 & 2500 & 0.2 & $/$ & 1 \\
Foam concrete & 40 & 600 & 0.21 & 0.3 & 15 \\
Medium sand & 448 & 1950 & 0.1 & 38 \\
\hline
\end{tabular}

Note. $E$ = modulus of elasticity.

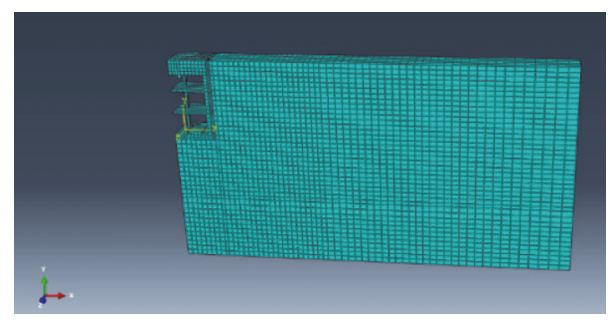

FIGURE 5: Three-dimensional model of Guofan Station.

$112.77 \mathrm{~m}$, and the longitudinal length of $8 \mathrm{~m}$ is established, as shown in Figure 5. The reason to take $8 \mathrm{~m}$ in longitudinal direction is that the longitudinal space of columns is $8 \mathrm{~m}$, and building a 3D model in the whole length of $113.77 \mathrm{~m}$ would make computation time too long to be accepted in the analysis.

Considering the whole calculation model, monitoring nodes were set from the position of underground diaphragm wall till the location $60 \mathrm{~m}$ away from the underground diaphragm wall, with an interval of $5 \mathrm{~m}$ roughly between two neighboring points.

\section{Calculation Results and Analysis}

4.1. Calculation Results of Cases 1, 2, and 3. As seen from Table 1, it is clear that case 1 has a low Young's Modulus of about $40 \mathrm{MPa}$, a low damping of about 0.05 , and a Poisson's ratio of 0.21 . This case represented a relatively soft foam concrete. By monitoring the time domain and frequency domain results of the point, the acceleration level of each monitoring point can be calculated according to the vibration evaluation method. The relationship between the acceleration level and the distance from the vibration epicenter under the condition of case 1 is shown in Figure 6.

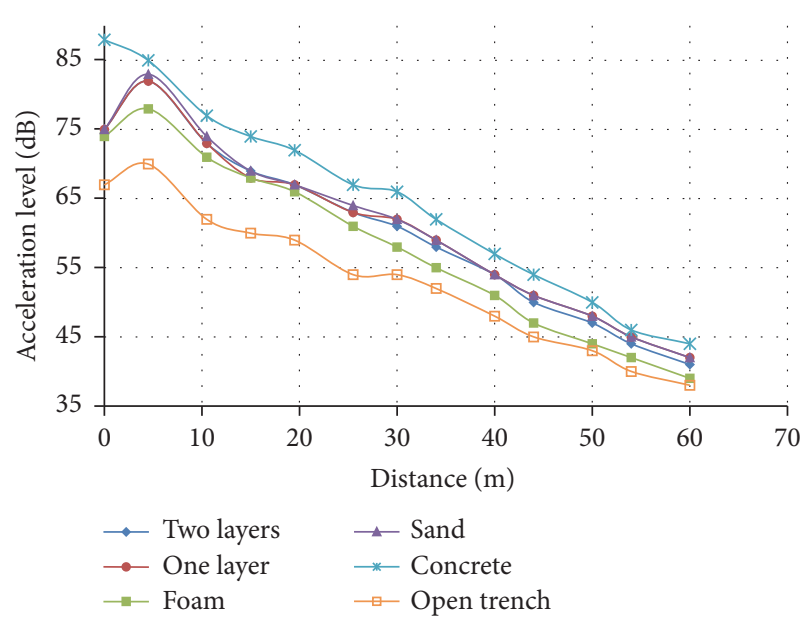

FIgURE 6: The relationship between the acceleration level and distance under case 1.

In order to facilitate the comparison among various results, Scheme 1 is renamed as one layer and Scheme 2 is renamed as two layers, while Schemes 3, 4, 5, and 6 are renamed as sand, foam concrete, concrete, and open trench, respectively. As seen from Figure 6, it is clear that vibration attenuates with the increase of distance. When the distance is about $60 \mathrm{~m}$ away from the vibration epicenter, the acceleration level of all the schemes falls below $45 \mathrm{~dB}$. Comparison between schemes of case 1 shows that concrete trench scheme has the biggest vibration and open trench cut the vibration most effectively. Foam concrete trench performs better than the other two schemes left, while two-layer scheme is slightly better than one-layer sand scheme in terms of vibration reduction.

The relationship between the acceleration level and the distance under case 2 is shown in Figure 7, which shows a 


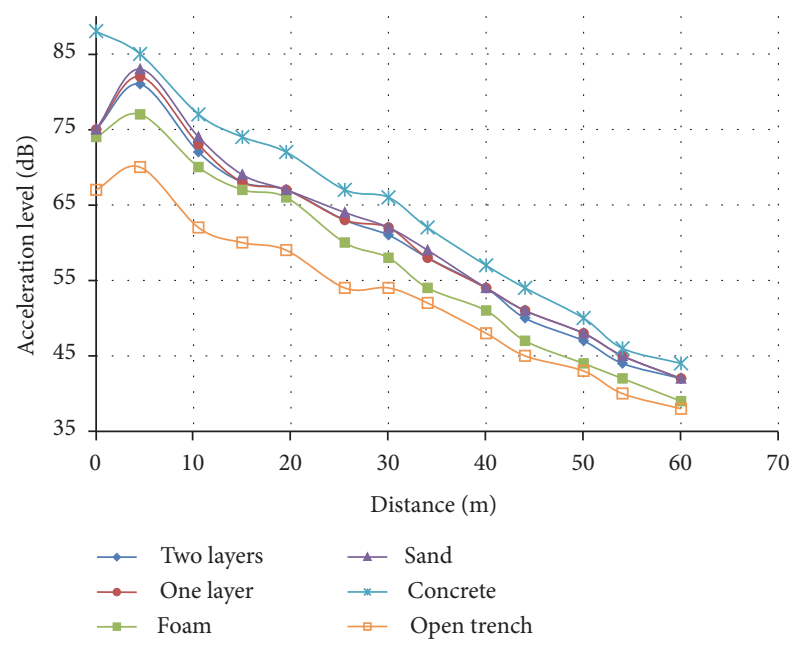

Figure 7: The relationship between the acceleration level and distance under case 2.

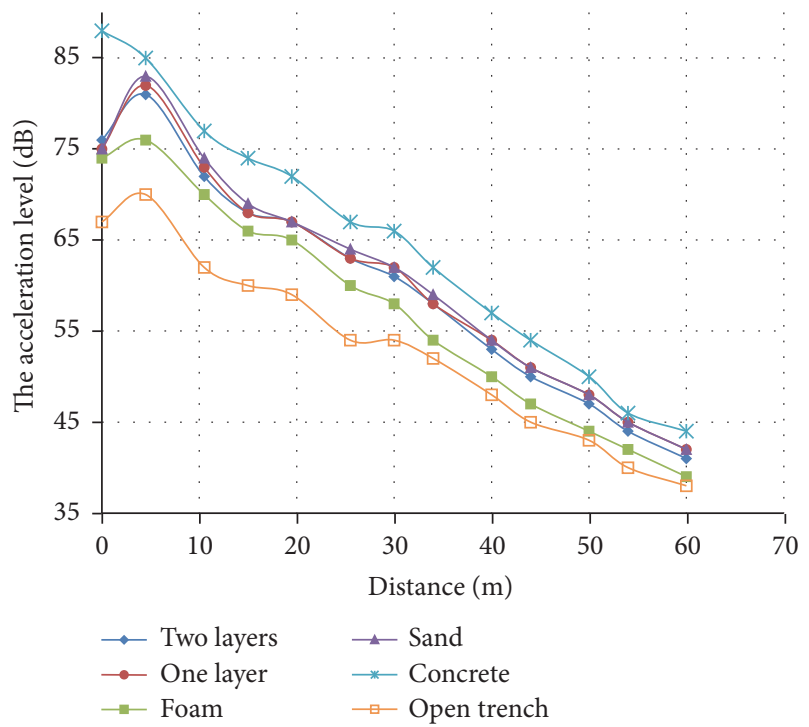

FIGURE 8: The relationship between the acceleration level and distance under case 3 .

similar trend in case 1 . This indicates that change of damping parameter of foam concrete did not change the calculation results much.

Figure 8 shows the relationship between the acceleration level and the distance from vibration epicenter under calculation case 3. Generally speaking, case 3 has a much bigger damping for foam concrete and thus has slightly more reduction of vibration especially for all foam concrete schemes.

4.2. Calculation Results of Case 4. The relationship between the acceleration level and the distance under the condition 4 is shown in Figure 9. Case 4 is slightly different from case 1 in that its Poisson's ratio is 0.31 , which is slightly larger than 0.21 in case 1 . While the general trend is the same as that in the above three cases, reduction degree seems to be bigger in

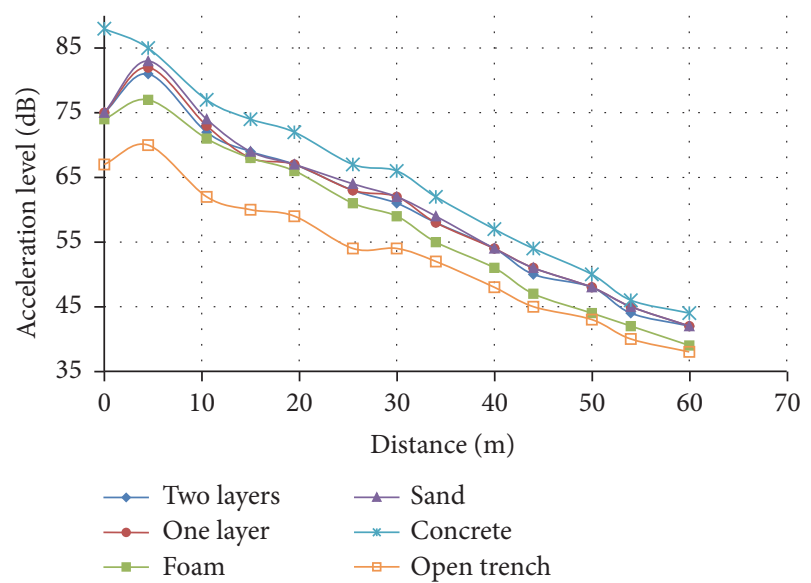

FIGURE 9: The relationship between the acceleration level and distance under case 4.

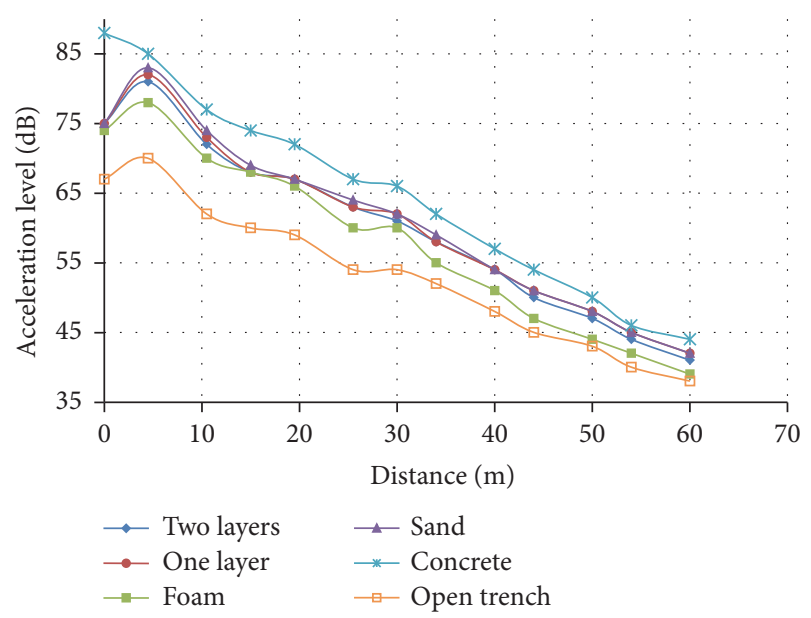

FIGURE 10: The relationship between the acceleration level and distance under case 5 .

case 4 than that in case 1 . The trend is that open trench has the highest effectiveness in reducing vibration, while trench filled with concrete has the highest acceleration level.

4.3. Numerical Result under Case 5. Case 5 (Figure 10) has the biggest Poisson's ratio for foam concrete, which is about 0.48 . However, the trend among different schemes is the same as other schemes. Schemes 1, 2, and 4 which represent one layer, two layers, and no layer of foam concrete performed almost the same during the construction.

4.4. Numerical Result under Case 6. The relationship between the acceleration level and the distance under case 6 is shown in Figure 11. This case is another extreme situation, where the foam concrete's elastic rigidity is 25 times higher than other schemes in order to observe the effects of Young's Modulus on vibration reduction. It is clear that, in this case, vibration reduction curve is almost the same as that of Schemes 1 and 2. It means that stiff foam concrete is not so good in reducing vibration. 


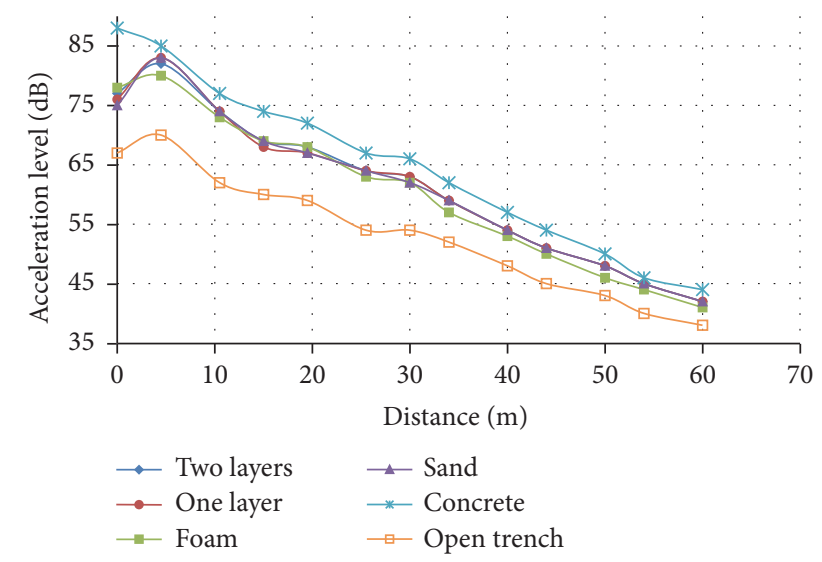

FIGURE 11: The relationship between the acceleration level and distance under case 6 .

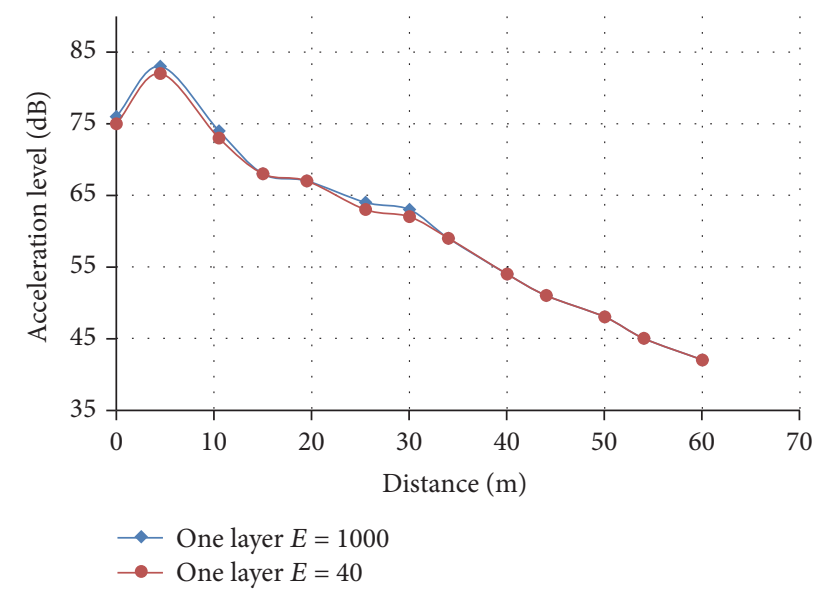

FIGURE 12: The comparison of acceleration between case 1 and case 6 in Scheme 1.

\subsection{Parameter Sensitivity Analysis}

4.5.1. Effect of Foam Concrete's Elasticity Modulus. The elasticity modulus of foam concrete is affected by the fabrication process, foaming agent, and other factors and will change within a larger range. Figures 12-14 checked the trends how the elasticity modulus of foam concrete influences the vibration reduction.

From Figures 12-14 it can be seen that the vibration reduction effect becomes lower as the elasticity modulus of the foamed concrete increases. This is because, with the increase of the elasticity modulus of the foam concrete, its wave impedance and wave impedance ratio both increase. The vibration reduction effect of different foamed concrete in Scheme 1 and Scheme 2 is slightly different, but there is a significant difference in Scheme 4. From Figures 6 and 11 , it can be seen that when the elasticity modulus of the foam concrete increases, the difference in vibration reduction effect in Schemes 1, 2, and 3 basically disappeared. Besides, the wave impedance of foam concrete is closer to that of sand, meaning that when wave impedance ratio of materials is close, its vibration reduction effect is quite similar.

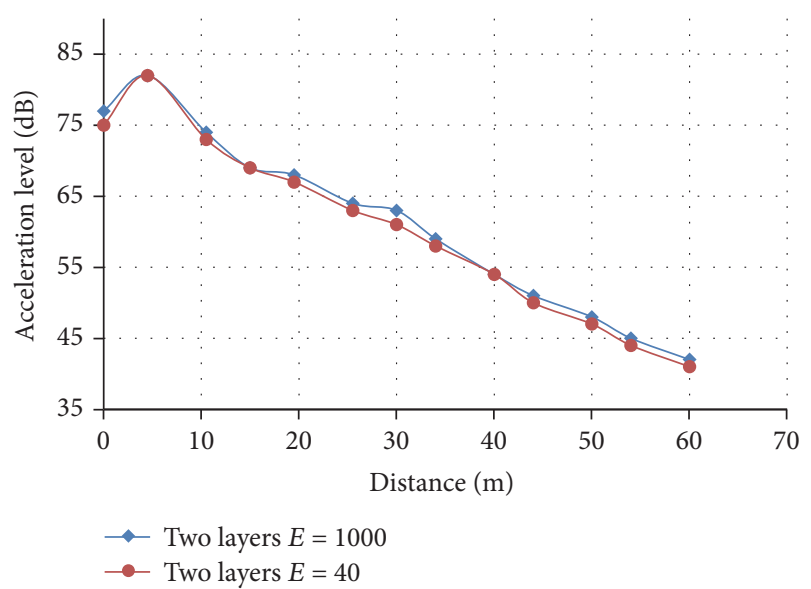

FIGURE 13: The comparison of acceleration level between case 1 and case 6 in Scheme 2.

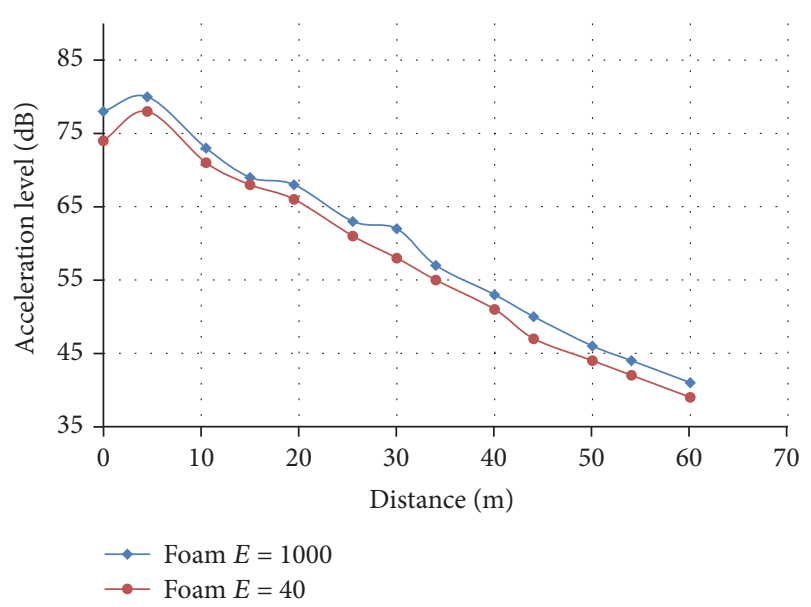

FIGURE 14: The comparison of acceleration between case 1 and case 6 in Scheme 4.

4.5.2. Effect of Foam Concrete's Poisson Ratio. In accordance with knowledge about wave impedance and wave impedance ratio, material's vibration reduction effect depends on these two parameters. The calculation of wave impedance is related to density, elasticity modulus, and Poisson Ratio of the material. The influence of the elasticity modulus of the concrete has been analyzed in the previous section. Since density of material can be uniquely determined, the effect degree of Poisson Ratio on the vibration reduction can thus be discussed here. The relationships between the acceleration level and the distance in Schemes 1, 2, and 4 under case 1, case 4, and case 5 are shown in Figures 15-17.

As can be seen from Figures 15-17, the change in Poisson's ratio of foam concrete has no significant effect on the vibration reduction effect in Schemes 1, 2, and 4. The wave impedance ratio under cases 1,2 , and 3 is very close, and the relation curve of acceleration level and the distance is basically coincident.

4.5.3. Effect of Foam Concrete's Damping Ratio. The effect of wave impedance ratio on the vibration reduction has been 


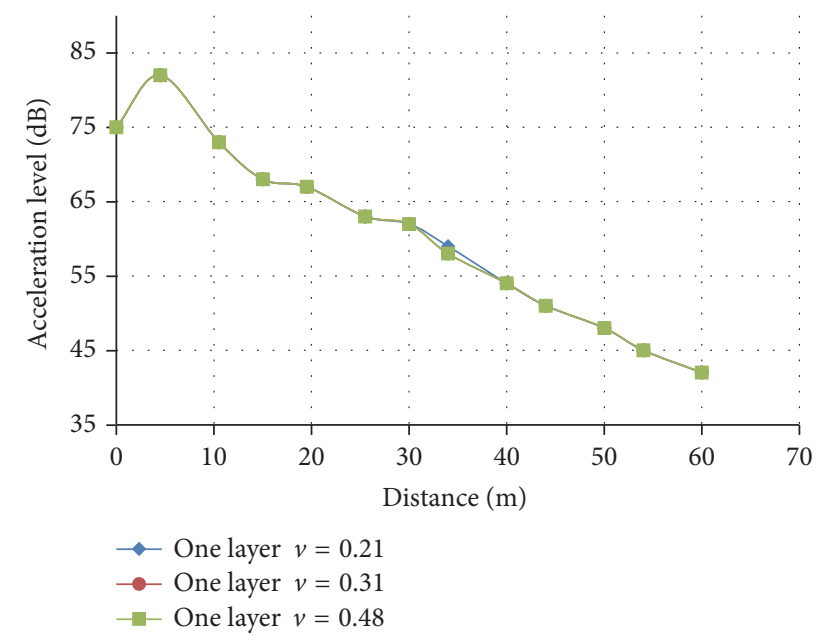

FIgURE 15: The comparison of acceleration level among cases 1, 4, and 5 in Scheme 1.

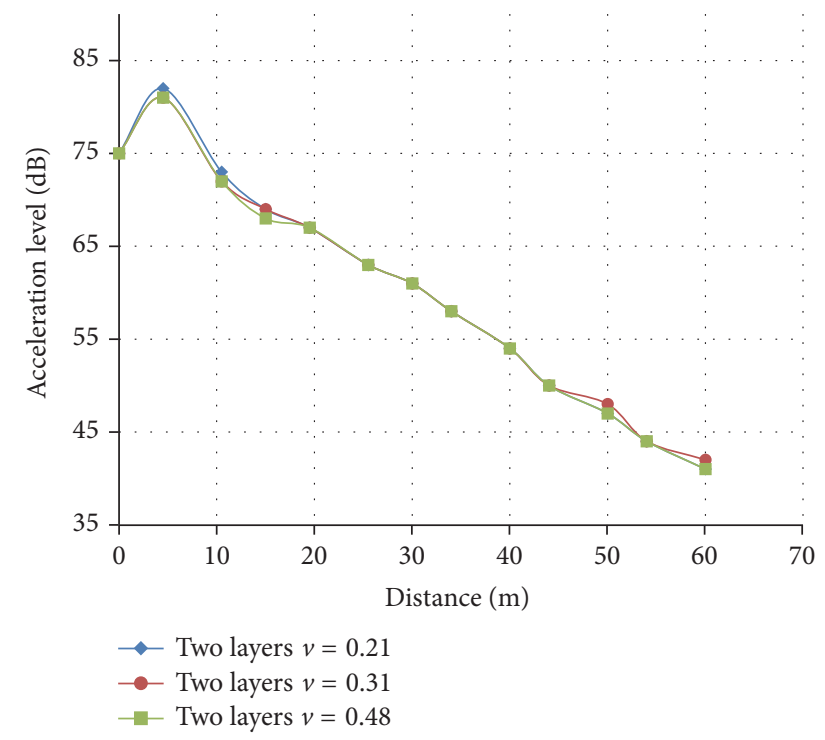

FIGURE 16: The comparison of acceleration among cases 1, 4, and 5 in Scheme 2.

analyzed in the previous two sections, and this section mainly examines the effect of damping ratio on the vibration reduction. Damping ratio of material is generally between 0 and 1 , where the damping ratio of the foam concrete has been set, respectively, to be $0.05,0.5$, and 0.95 , and the calculation results under cases 1, 2, and 3 are shown in Figures 18-20.

It can be seen from Figures 18-20 that, with the change of the damping ratio in Scheme 1 and Scheme 2, the relation curve of the acceleration level and distance has no significant difference, which means that the change of the damping ratio has little effects on vibration reduction. As for Scheme 4, the bigger the damping ratio of the foamed concrete, the better the vibration reduction effect, but the difference is not that large, which indicates that the damping ratio has only slight effect on the vibration reduction.

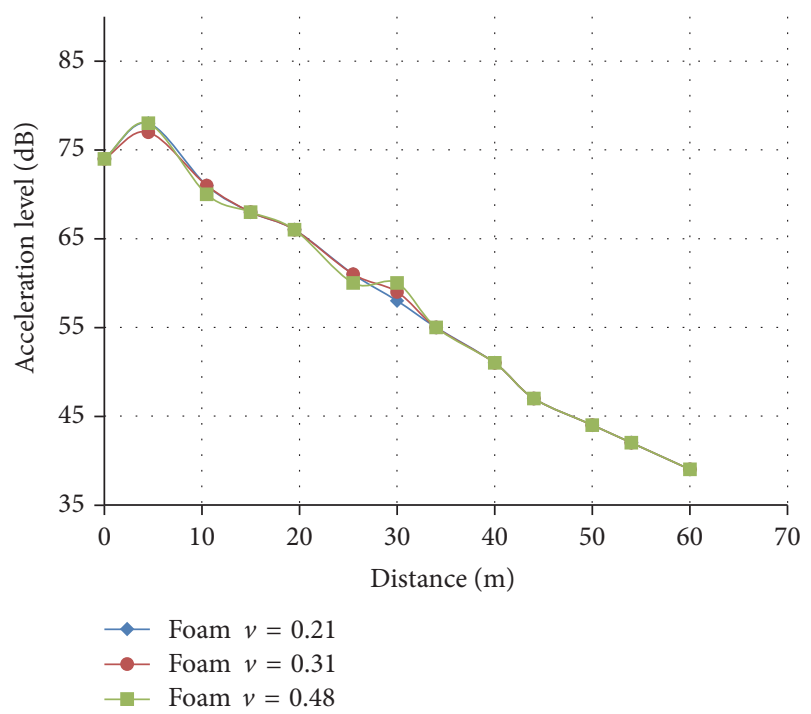

FIgURE 17: The comparison of acceleration level among cases 1, 4, and 5 in Scheme 4.

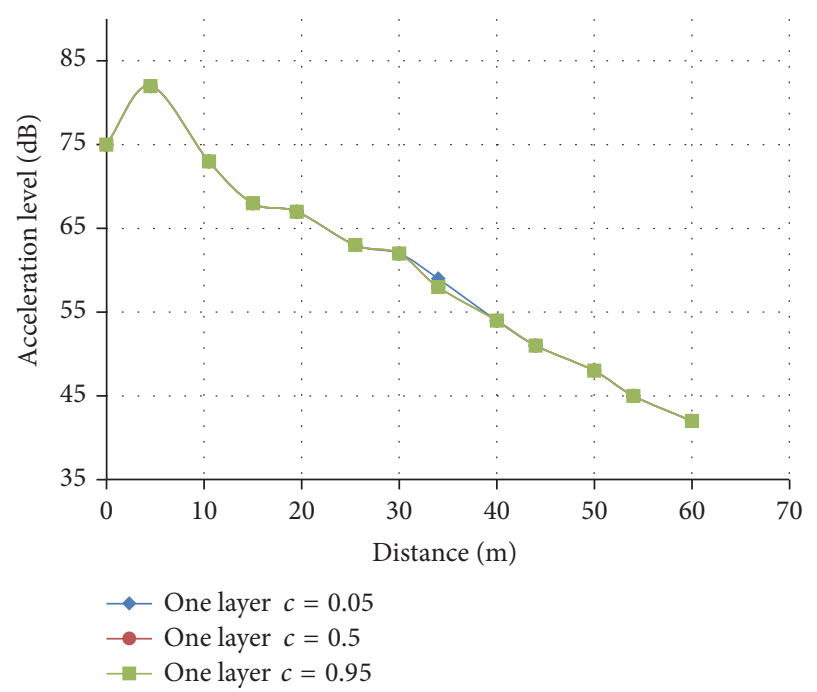

FIgURE 18: The comparison of acceleration level among cases 1, 2, and 3 in scheme 1.

It can be seen from the relation curve of acceleration level and distance from vibration epicenter in Figures 6-10 that, for Guofan Road station whose structure is surrounded by separated retaining wall, the vibration can be reduced by the trench between station structure and underground diaphragm wall, and the reduction effect is best with an open trench. The vibration reduction effect of the trench filled with foam concrete is obviously better than the trench filled with concrete; namely, Scheme 4 is obviously better than Scheme 5. The greatest reduction of acceleration level of the underground diaphragm wall is up to $14 \mathrm{~dB}$, the average is about $8 \mathrm{~dB}$, and the acceleration level decay is $9.4 \%$. In addition, the vibration reduction effect in Scheme 1, Scheme 2 , and Scheme 3 is better than that of Scheme 5 but worse than that of Scheme 4. The vibration reduction effect in Scheme 


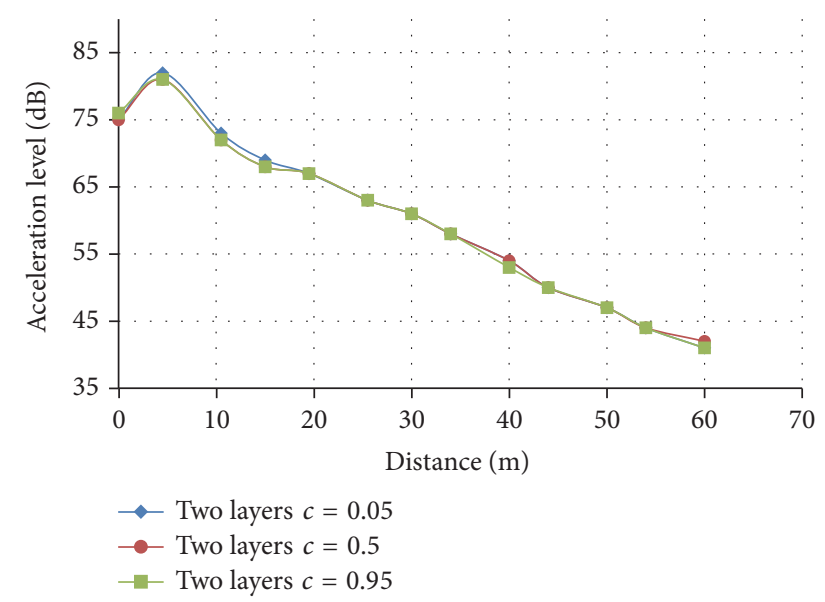

FIGURE 19: The comparison of acceleration level among conditions 1,2 , and 3 in Scheme 2.

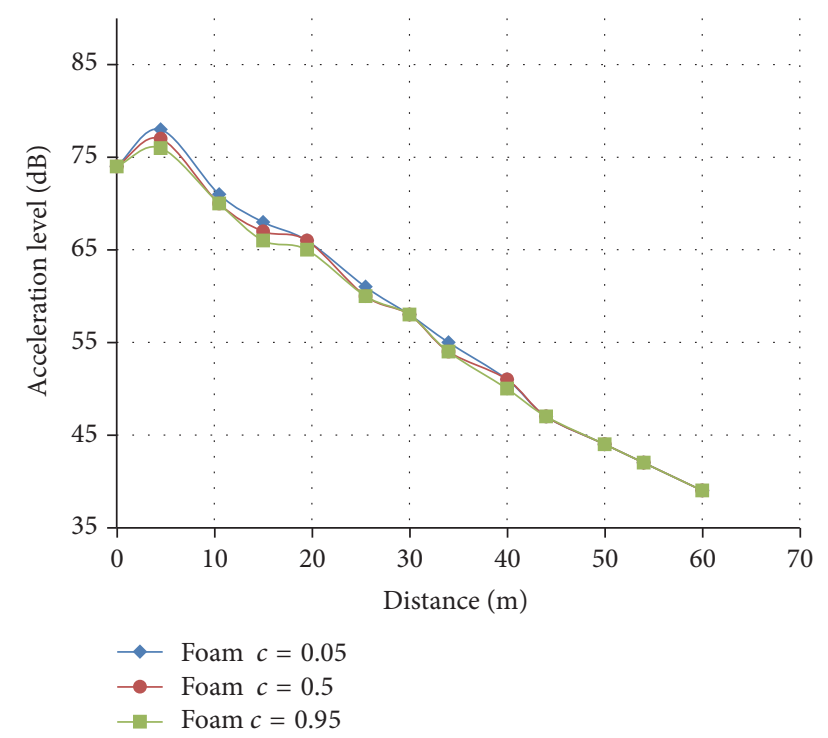

FIGURE 20: Comparison of acceleration level among cases 1, 2, and 3 in Scheme 4.

2 is slightly better than that in Scheme 1, and the vibration reduction effect in Scheme 1 is slightly better than Scheme 3, with about $2 \mathrm{~dB}$ decrement of acceleration level.

For this project, by filling vibration reduction material into the gap trench between station structure and underground diaphragm wall, vibration reduction and separation can be achieved. Several materials have been checked on their effect of reducing vibration and different parameters were considered. In this case where the gap trench is surrounded by concrete which has large wave impedance, filling in the trench with foam concrete which has relatively small wave impedance is most effective in reducing and separating vibration from the ground. In this meaning, Scheme 4 is the best filling scheme and can achieve a good vibration isolation and reduction effect. However, considering the balance between vibration reduction effect and the cost or construction feasibility, one-layer scheme (Scheme 1) turned out to be the best one.

Based on the above analysis, Scheme 1 has been adopted in the design and construction work for Guofan Road station project. Further field measurement data is highly expected after the service start.

\section{Conclusion}

In this paper, the vibration separation and reduction effect of compound materials filled in a trench has been discussed in the scope of Guofan Road station in Shanghai Metro Line 10 , phase two project. Foam concrete and sand materials are considered as barrier materials. Elasticity modulus, Poisson's ratio, and damping ratio of foam concrete are taken as sensitive parameters influencing the reduction effect, and series of numerical analysis have been conducted on different conditions to analyze their vibration reduction effect. The conclusions are as follows:

(1) Separation trench surrounding subway station structures is effective in separating vibration of the station from adjacent environment. Foam concrete and sand can act as barrier materials.

(2) In this project, when elasticity modulus, Poisson ratio, or wave impedance ratio of the foam concrete increases, the vibration reduction effect is lowered.

(3) The changes of Poisson ratio show little influence on foam concrete's wave impedance and vibration reduction.

The damping ratio of foam concrete has an impact on vibration reduction but the impact is not obvious.

(4) The barrier should be composed of a material with significant vibration reduction effect. When the barrier consists of two kinds of materials with different vibration reduction effect, the reduction effect depends on the material with the lower vibration reduction effect, and the combined effect will be obviously lowered.

\section{Conflicts of Interest}

The authors declare that they have no conflicts of interest.

\section{References}

[1] D. D. Barkan and L. Drashevska, Dynamics of bases and foundations, McGraw-Hill, 1960.

[2] D. Woods R, "Screening of Surface Wave in Soils," Journal of the Soil Mechanics Foundations Division, pp. 94-951, 1968.

[3] F. E. Richart, Vibrations of Soils and Foundations, China Architecture Building Press, 1976.

[4] W. A. Haupt, "Model tests on screening of surface waves," in Proceedings of the 10th international conference on soil mechanics and foundation engineering, vol. 3, pp. 215-222, 1981.

[5] A. Haupt W, "Surface waves in nonhomogeneous half-space," Dynamical methods in soil and rock mechanics, pp. 335-367, 1978. 
[6] W. A. Haupt, "Numerical methods for the computation of steady-state harmonic wave fields," Proc. of DMSR, vol. 1978, no. 77, pp. 255-280, 2004.

[7] G. Schmid, N. Chouw, and R. Le, "Shielding of structures from soil vibrations," Soil Dynamics And Earthquake Engineering V, pp. 651-662, 1991.

[8] A. T. Peplow, C. J. C. Jones, and M. Petyt, "Surface vibration propagation over a layered elastic half-space with an inclusion," Applied Acoustics, vol. 56, no. 4, pp. 283-296, 1999.

[9] H. Takemiya and F. Chen, "Enhanced seismic design of a pile foundation by cell-type wib," Doboku Gakkai Ronbunshuu A, vol. 62, no. 2, pp. 467-477, 2006.

[10] C. Qiu, G.-Y. Gao, Z.-Q. Yue et al., "Research on the failure mechanism of barrier for isolating vibration," Northwestern Seismological Journal, vol. 25, no. 3, pp. 198-203, 2003.

[11] L. S. Li, Q.-L. Xie, and L. Tang, "Influence Factors of Impact Reduction Performance of Foamed Concrete Backfill Layer," Blasting, vol. 03, pp. 166-171, 2015.

[12] D. F. Zhao, W. W. Kong, and X. R. Song, "Testing method of elastic modulus of foamed concrete," Concrete, vol. 9, pp. 4-6, 2008.

[13] X. Z. Yan and Q. F. Yao, "Experimental Study On The Mechanical Propertyof Foamed Concrete," Industrial Construction, vol. zl, pp. 962-964, 2007.

[14] L. Qiu-Huan, L. Fang-yun, and W. Xiang-yun, "Study On Regulation Of Stress Wave Propagation In Rockmass With Compound Structures," Chinese Journal of Rock Mechanics and Engineering, vol. 22, no. 11, pp. 1832-1836, 2003.

[15] Y.-K. Shen, Study on The Propagation Laws of Subway-induced Vibration and Isolation of Reduction Methods of Build Vibration, Tongji University, 2007.

[16] J. L. Ma and H. P. Ding, "Comparative study on influence of different damping values in soil-layer seismic response analysis," Journal of Disaster Prevention and Mitigation Engineering, pp. 517-523, 2013.

[17] Y. Gu, J.-B. Liu, and Y.-X. Du, "3D consistent viscous-spring artificial boundary and viscous-spring boundary element," Journal of Engineering Mechanics, vol. 24, no. 12, pp. 31-37, 2007. 


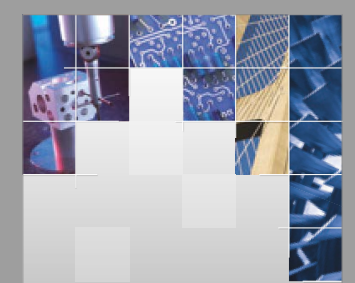

\section{Enfincering}
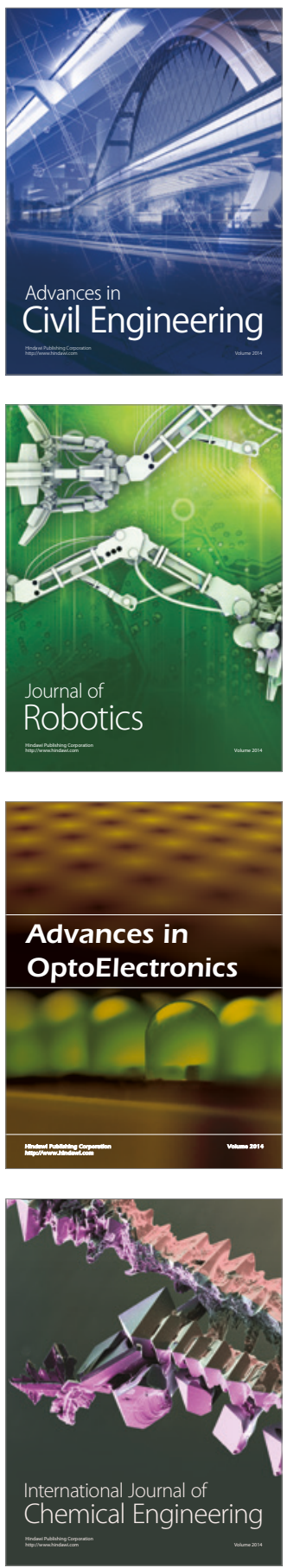

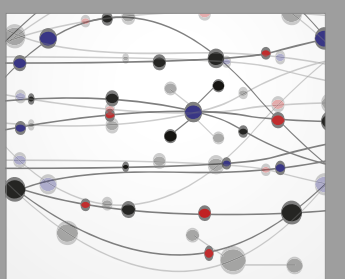

The Scientific World Journal

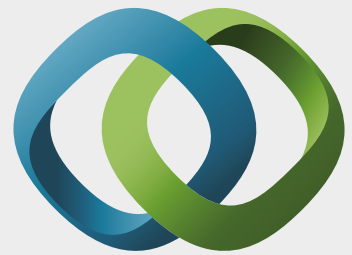

\section{Hindawi}

Submit your manuscripts at

https://www.hindawi.com
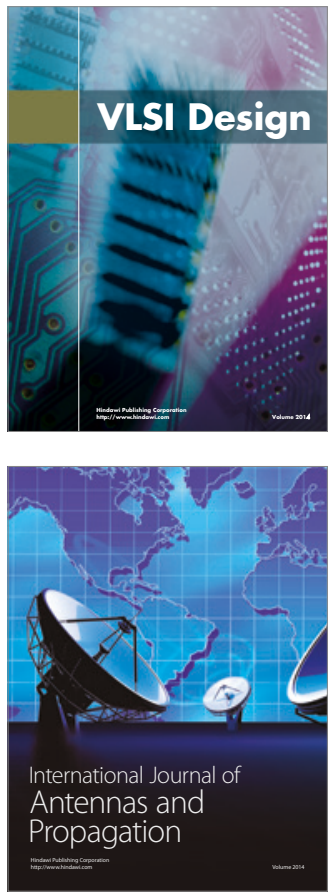

\section{Rotating}

Machinery
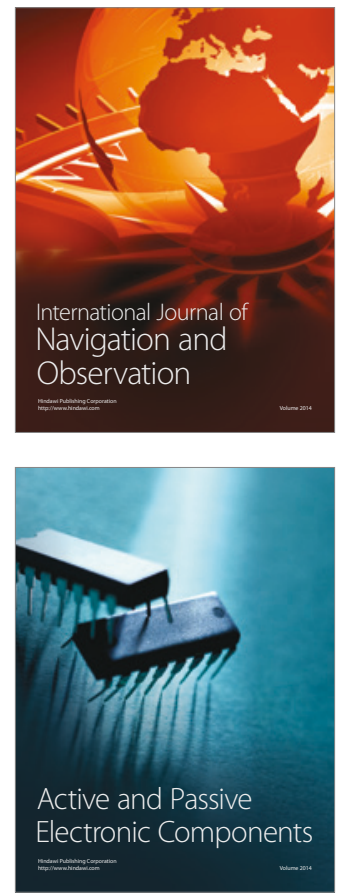
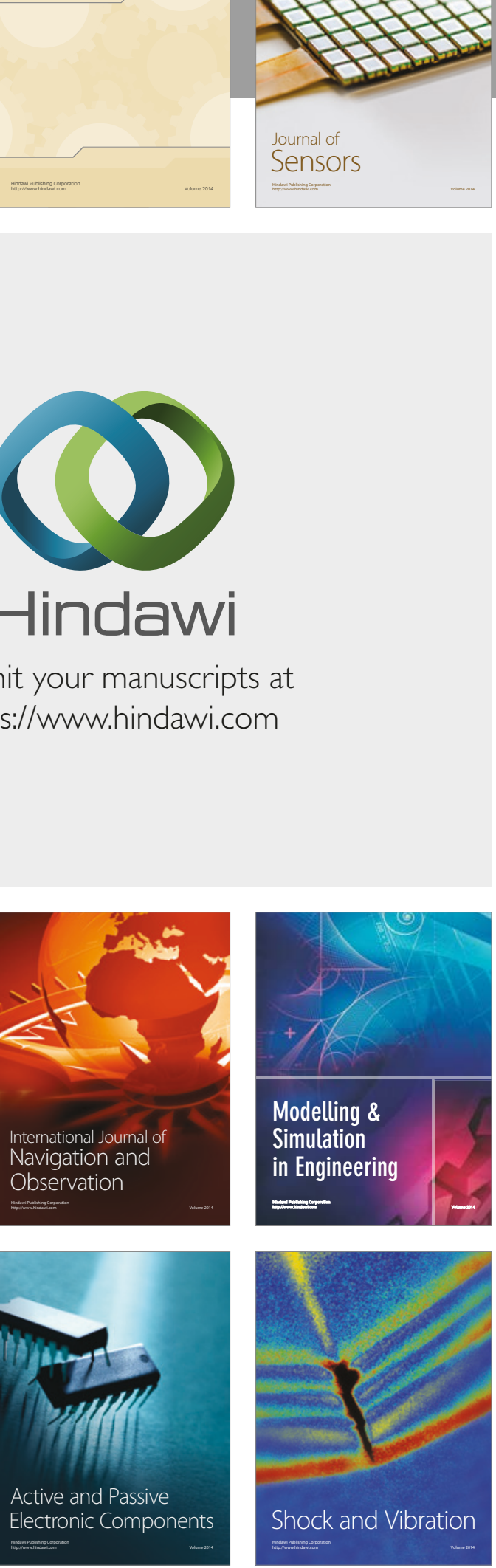
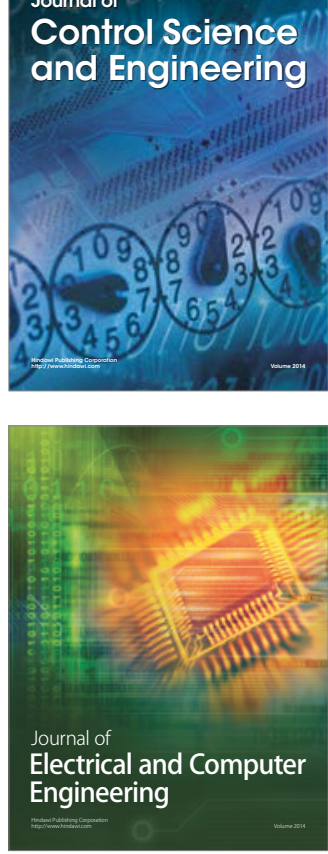

Distributed

Journal of

Control Science

and Engineering
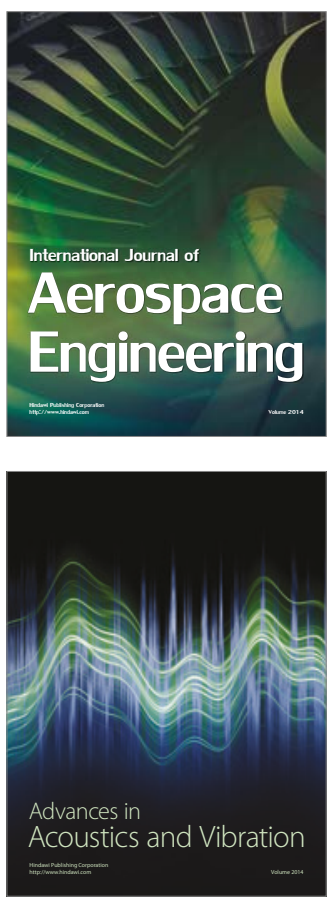

Sensor Networks 